\title{
Post-Covid Renal Mucormycosis: A Case Report
}

\author{
B. Madhumitha ${ }^{1}$ Ganesh Rajagopal ${ }^{1} \quad$ N. Karunakaran ${ }^{1} \quad$ T. Mukuntharajan $^{1}{ }^{10}$
}

${ }^{1}$ Department of Imaging and Interventional Radiology, Meenakshi Mission Hospital and Research Centre, Madurai, Tamil Nadu, India

J Gastrointestinal Abdominal Radiol ISGAR 2022;5:70-74.
Address for correspondence Madhumitha B., MBBS, DNB, Department of Imaging and Interventional Radiology, Meenakshi Mission Hospital and Research Centre, Lake area, Melur Main Road, Madurai, Tamil Nadu 625107, India (e-mail: madhu123ind@gmail.com).

\author{
Abstract \\ Keywords \\ - COVID-19 \\ - diabetes \\ - renal mucormycosis
}

The Pandora's box has been opened in the twenty-first century unleashing "the Coronavirus." There has been an increase in the incidence of mucormycosis during this coronavirus disease 2019 (COVID-19) pandemic with many case reports of rhinoorbito-cerebral and pulmonary mucormycosis. Diabetes mellitus, COVID, and Mucor fungus together are a dangerous trio associated with high mortality. We report a rare case of renal mucormycosis following COVID pneumonia in a 64-year-old patient with uncontrolled diabetes; with the imaging findings of left emphysematous pyelonephritis and cystitis, nonfunctioning left kidney, and left main renal artery and vein thrombosis. This is the first such case reported to our knowledge. A high degree of clinical suspicion with prompt recognition of imaging findings is essential to improve the chances of survival in such patients.

\section{Introduction}

The coronavirus disease 2019 (COVID-19) pandemic continues despite affecting more than 196 million cases and claiming more than 4.2 million lives globally. ${ }^{1}$ Adding to the disease burden, we also have incidences of opportunistic fungal infections including aspergillosis, candidiasis, and mucormycosis in patients either after recovery or during the resolving phase of COVID-19 pneumonia. ${ }^{2}$ There has been a surge of the COVID-19-associated mucormycosis (CAM) especially in India. ${ }^{3}$ The various presentations of mucormycosis in decreasing order of frequency include oculo-rhino-cerebral, pulmonary, gastrointestinal, cutaneous, and disseminated mucormycosis. ${ }^{4}$ There has been a case report of fatal primary renal mucormycosis in a post-COVID young male with no prior risk factors, with the imaging finding of renal infarct; the prior history of COVID pneumonia could have been coincidental rather than contributory in that case. ${ }^{5}$ There has been another case report of renal mucormycosis with a nonfunctioning kidney and main renal artery thrombosis in a post-COVID patient; much similar to our case. ${ }^{6}$ Till date there have been no reports of emphysematous pyelonephritis with main renal artery and vein thrombosis; an aggressive presentation of renal mucormycosis in a post-COVID individual. Here, we report one such rare case.

\section{Case Report}

A 64-year-old male patient presented to the emergency department with complaints of abdominal pain, dysuria, hematuria, and decreased urine output for 1 week. He was a known diabetic and hypertensive on oral hypoglycemic agents and antihypertensives for the past 10 years. There were no presenting complaints of fever, breathing difficulty, or loss of consciousness. There was history of fever 1 month back with a positive reverse transcription polymerase chain reaction test for COVID-19; he was treated at an outside published online January 24, 2022
DOI https://doi.org/ 10.1055/s-0041-1740478. ISSN 2581-9933.

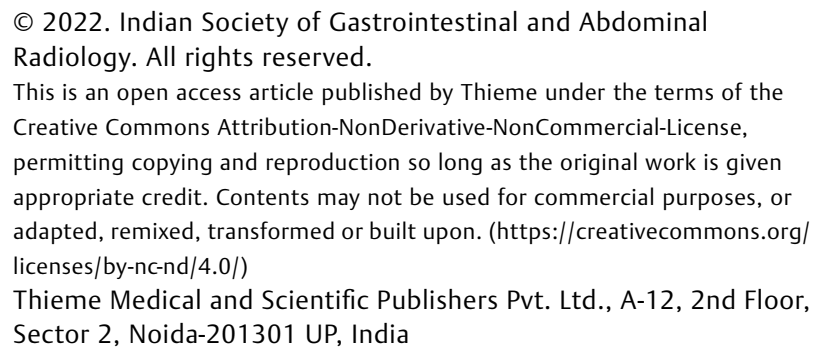


hospital and discharged following which he was apparently asymptomatic till a week back, when he developed the abovementioned symptoms and was referred to our hospital for further management. On examination, the patient was tachypneic and afebrile; blood pressure and oxygen saturation were within normal limits. Diffuse abdominal tenderness more over bilateral loin region was noted.

Laboratory examination revealed normal serum creatinine $(0.9 \mathrm{mg} / \mathrm{dL})$ initially and an increased total leukocyte count $\left(23,000\right.$ cells $\left./ \mathrm{mm}^{3}\right)$ with neutrophilia (94\%) and severe lymphopenia (2\%). There was elevated erythrocyte sedimentation rate $(118 \mathrm{~mm} / \mathrm{h})$, C-reactive protein $(26.6 \mathrm{mg} / \mathrm{dL})$, Ddimer $(1,670 \mathrm{ng} / \mathrm{mL})$, and procalcitonin $(6.2 \mathrm{ng} / \mathrm{mL})$ levels pointing toward sepsis. The patient also had poor glycemic control with increased random blood sugar $(253 \mathrm{mg} / \mathrm{dL})$, serum ketones $(28.0 \mathrm{mg} / \mathrm{dL})$, and glycated hemoglobin levels (11.7\%). Urine analysis showed few pus cells and red blood cells on microscopy; no fungal elements were seen. Urine dipstick reflectance photometry detected protein, blood, and glucose but was negative for ketones in urine. There was no growth in aerobic bacterial culture from urine sample.

An unenhanced computed tomography (CT) of the patient's abdomen and pelvis was performed using 128 slice Multidetector CT-Optima 660 (GE Healthcare, Milwaukee, Wisconsin, United States); following which $100 \mathrm{~mL}$ of iodinated contrast Omnipaque (GE Healthcare, Shanghai, China) was administered at a flow rate of $4 \mathrm{~mL} / \mathrm{s}$ using a pressure injector. Images with a slice thickness of $5 \mathrm{~mm}$ and $0.625 \mathrm{~mm}$ reconstruction in the nephrogenic, excretory, and delayed phase were obtained at a time-delay of 55 seconds, 90 seconds, and 5 minutes, respectively. The study revealed an enlarged left kidney with perinephric fat stranding, thickening of Gerota's fascia, and multiple air pockets in the left kidney, left upper ureter, urinary bladder, and surrounding soft tissues ( $\mathbf{- F i g s .} \mathbf{1}$ and $\mathbf{2}$ ). There was complete absence of contrast enhancement in the left renal parenchyma ( - Fig. 3) with thrombosis of the left main renal artery ( - Fig. 4) and thrombosis with air foci within the left main renal vein (-Fig. 5). No evidence of extension of thrombus into the great vessels noted. There were no renal or perinephric abscess/collections. There was no excretion of contrast into

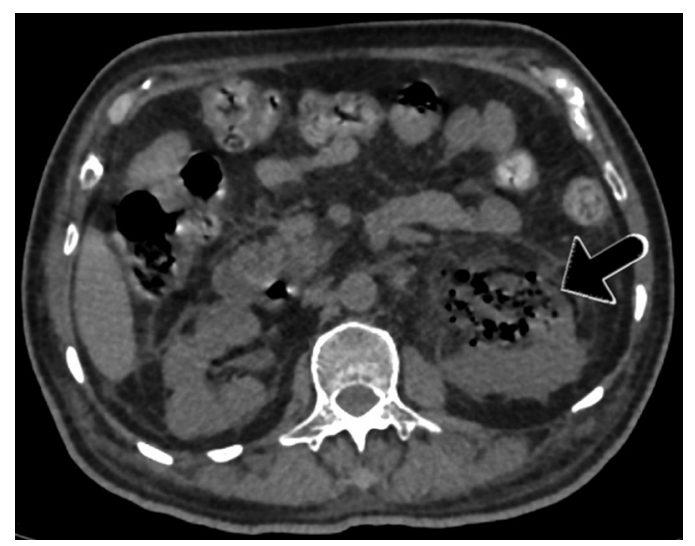

Fig. 1 Axial noncontrast-enhanced computed tomography image showing the bulky left kidney with air pockets and extensive surrounding perinephric fat stranding (arrow).

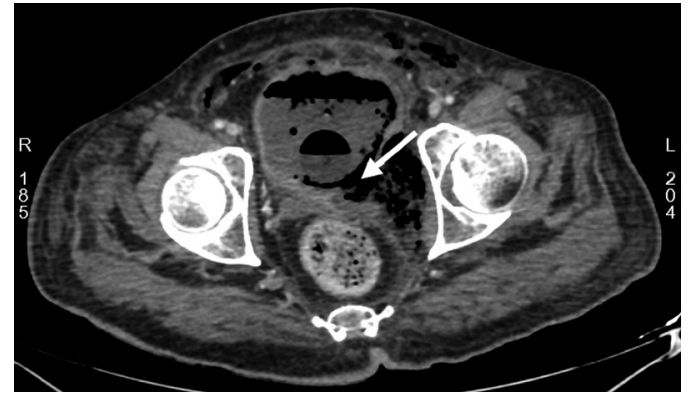

Fig. 2 Axial contrast-enhanced computed tomography image showing the partially filled urinary bladder with Foley's bulb in situ and extensive air pockets in the bladder wall mainly the left lateral wall (arrow) extending into the surrounding soft tissues.

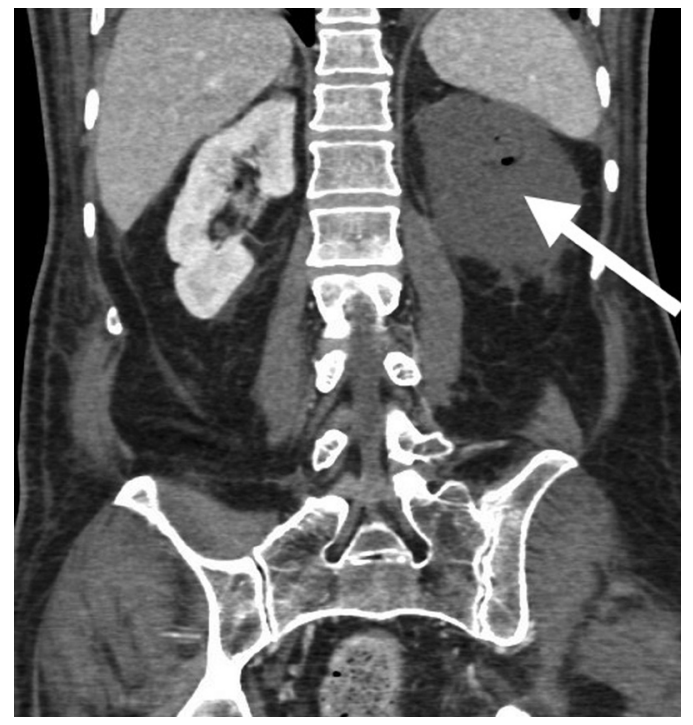

Fig. 3 Coronal reformatted contrast-enhanced computed tomography image showing complete absence of contrast enhancement of left kidney with air pockets in renal parenchyma (arrow).

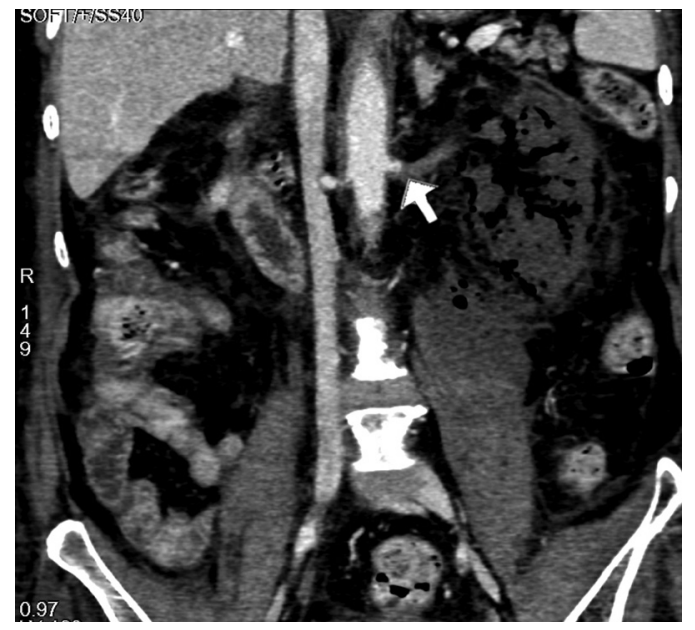

Fig. 4 Coronal reformatted contrast-enhanced computed tomography image showing complete thrombosis of left main renal artery (arrow). 


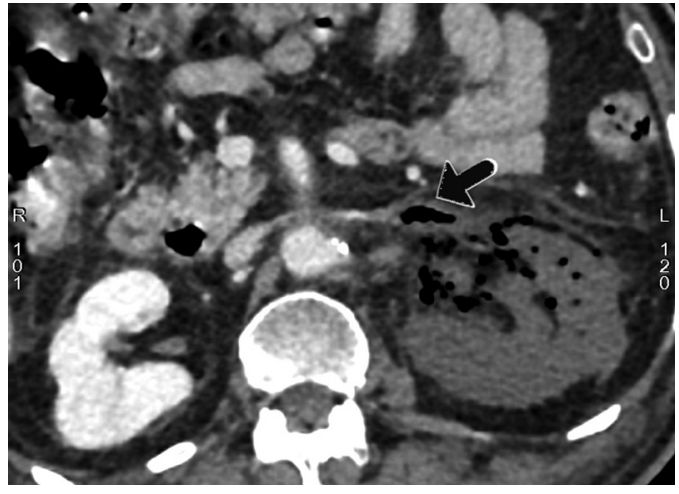

Fig. 5 Axial contrast-enhanced computed tomography image showing thrombosis of left main renal vein with air foci within the thrombus (arrow), inferior vena cava appears normal.

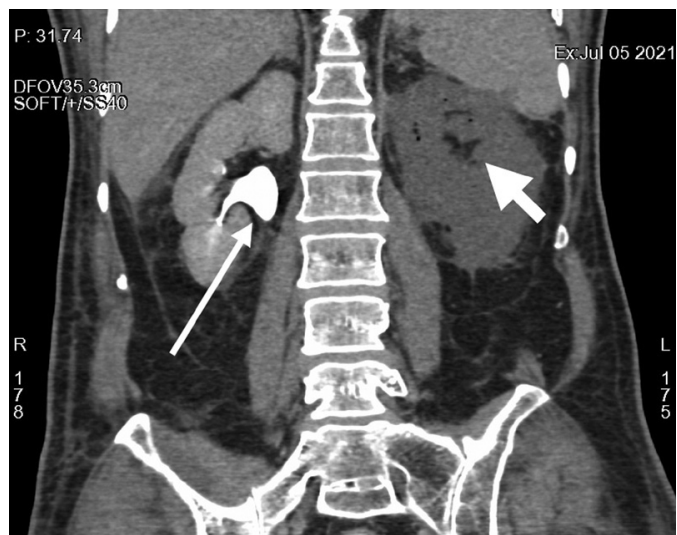

Fig. 6 Coronal reformatted contrast-enhanced computed tomography delayed phase image showing normal excretion of contrast into the right pelvicalyceal system (long thin arrow) and no excretion of contrast in the left pelvicalyceal system (short broad arrow).

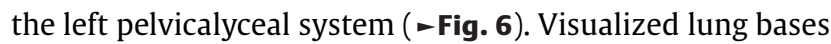
showed an area of consolidation with central ground-glass opacity (atoll sign) and cavitation in left lower lobe and bilateral minimal pleural effusion ( - Fig. 7A and B). A possibility of renal mucormycosis with superadded anaerobic infection was considered.

Cystoscopy was done under general anesthesia which revealed a pus-filled bladder and multiple bladder washes were given. The right ureteric orifice was identified and a double-J stent was placed in the right-side collecting system. The left ureteric orifice could not be identified. The patient was then explored through a left flank cutting incision; left nephrectomy along with removal of left upper ureter and adrenal gland was performed with multiple washes and closed. Gross examination revealed the entirely necrosed left kidney with surrounding fat saponification ( - Fig. 8). The histopathological examination ( $\mathbf{- F i g s . 9} \mathbf{9}, \mathbf{1 0}, \mathbf{1 1}$ ) showed the necrotic renal parenchyma with neutrophilic infiltration and fungal hyphae with evidence of angioinvasion. The hyphae were aseptate, ribbon-like with a large diameter, irregular width, and were branching at right angles. The left ureter and adrenal gland were not involved.

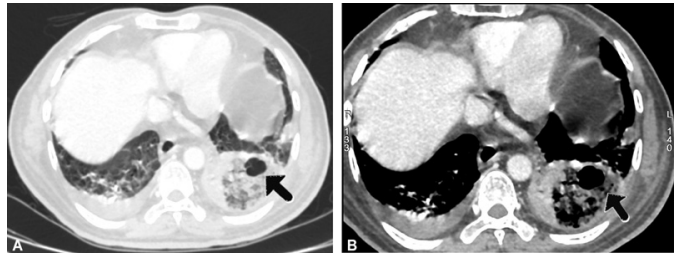

Fig. 7 Axial contrast-enhanced computed tomography images of the lung bases in lung window (A) and mediastinal window (B) showing bilateral minimal pleural effusion and areas of consolidation with atoll sign and cavity (arrow) in left lower lobe.

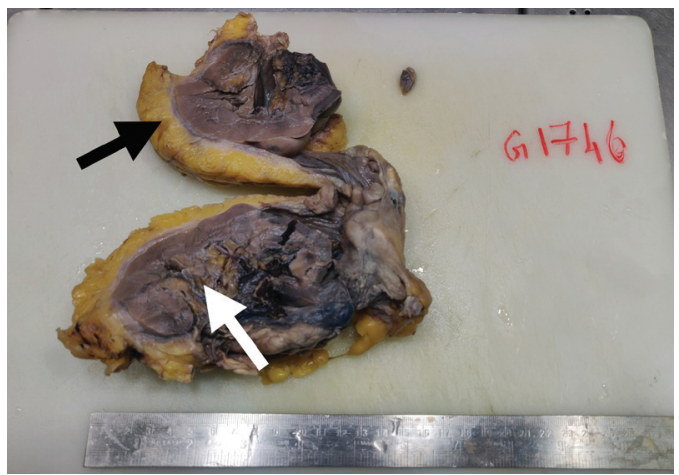

Fig. 8 Cut surface of the enlarged left kidney showing extensive areas of blackish discoloration (white arrow) and surrounding fat saponification (black arrow).

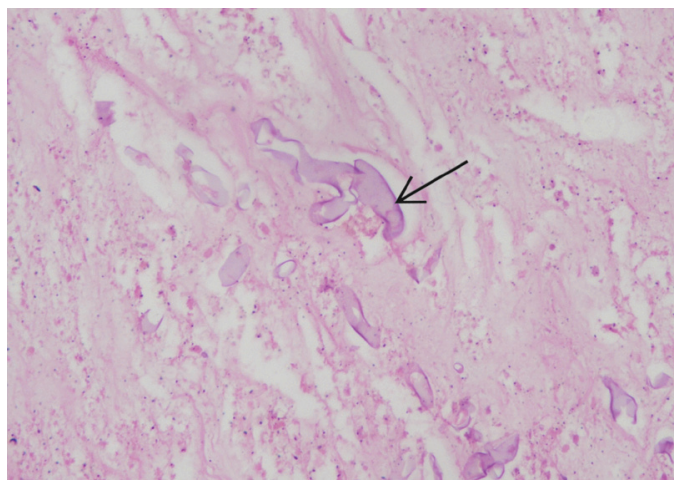

Fig. 9 Renal parenchyma showing extensive coagulative necrosis with neutrophilic infiltration and fungal hyphae (thin long arrow). Hematoxylin and eosin stain, $\times 400$.

Despite treatment, the sepsis worsened and the patient continued to deteriorate. The attenders wished to continue his treatment elsewhere and got discharged against medical advice and had succumbed to the disease in a week.

\section{Discussion}

Mucormycosis is caused by a group of angioinvasive fungi under the order Mucorales; the most common species involved are the Rhizopus and Mucor. ${ }^{7}$ Various risk factors are known to be associated, the most common being 


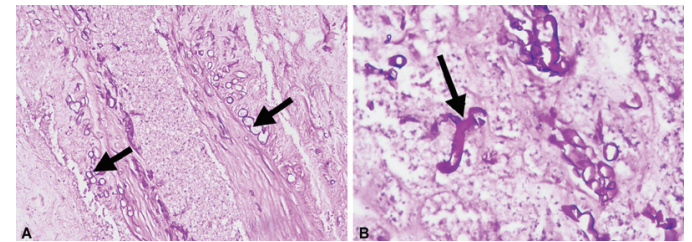

Fig. 10 Pleomorphic, ribbon-like, broad and aseptate fungal hyphae (arrows) seen along the vessel wall (periodic acid-Schiff stain, $\times 100$ ) (A) and in the necrotic renal parenchyma (periodic acid-Schiff stain, $\times 400)(B)$.

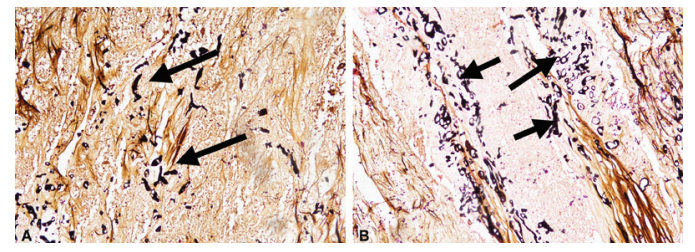

Fig. 11 Fungal hyphae seen in the renal parenchyma (arrows in A) and seen invading the blood vessel (arrows in B) (Gomori methenamine silver stain, $\times 100$ ).

uncontrolled diabetes mellitus; others include hematological malignancy, stem cell or organ transplant recipients, neutropenia, desferrioxamine therapy, and corticosteroid use. ${ }^{8,9}$ An increase in the incidence of CAM is attributed to the widespread use of steroids, hypoxemia, prolonged hospitalization needing intensive care, and previously immunocompromised individuals; but cases have also been reported in immunocompetent individuals with no prior risk factors. ${ }^{10}$ The COVID upregulates the glucose receptor protein GRP-78 which helps in the penetration and damage of endothelial cells by Mucor fungi leading to angioinvasion. ${ }^{11}$ Glucose, iron, and $\beta$-hydroxybutyrate enhance the fungal growth. ${ }^{12}$ The COVID-associated inflammation or its treatment modalities might contribute to the increase in these metabolites predisposing the individual to fungal infections. $^{3}$ There might exist a deeper pathophysiological link between the two pathogens that waits to be unraveled yet.

Phagocytes (neutrophils) are the first line of body's defense against fungal pathogens. These white blood cells engulf them in a process called phagocytosis. Sometimes a reverse process occurs, called the "vomocytosis," in which the pathogen is ejected back out of the cell without the lysis of either the host cell or the pathogen. There has been no study regarding the demonstration of vomocytosis with Mucor fungi yet. There has been a study which showed viral coinfection can enhance the rate of vomocytosis of fungus by macrophages. ${ }^{13}$ We hypothesize that COVID coinfection could enhance the vomocytosis of Mucor by macrophages and when at the wrong time and wrong place be catastrophic for the patient. COVID has altered the immune system of our patient predisposing him to fungal infection; the lungs were already affected by the COVID, hence the primary site of inoculation could have been the lung and being an uncontrolled diabetic with ketoacidosis provided a favorable environment for the growth of fungi; the Mucor could have then reached the kidney with the help of phagocytosis and vomocytosis by the neutrophils.

Ultrasonography remains the first line of investigation and can show the enlarged echogenic kidneys with hypoechoic areas of abscesses, perinephric fluid collections, hydronephrosis, and cystitis. ${ }^{14}$ CT features include diffuse patchy nephrogram, inhomogeneous enhancement with areas of low attenuation, perinephric fluid collections, and no contrast excretion. ${ }^{15}$ There can be multiple hypodense nonenhancing areas (intrarenal segmental infarcts) or even complete absence of contrast enhancement of the kidney. There can be thrombosis of the main renal artery and vein, but are usually spared with thrombosis of intrarenal segmental branches leading to infarction, which can be demonstrated on Doppler sonography. Magnetic resonance imaging study will show hypointense signal on T1-weighted imaging (WI) and hypointense signal on T2WI with diffusion restriction; in contrast to other infections which will be hypertintense on T2WI with diffusion restriction owing to the edema and inflammation. ${ }^{16}$ This typical T2 hypointensity with diffusion restriction seen in mucormycosis might be attributed to the completely infarcted tissue with necrotic debris and fungal filled abscesses with high iron (paramagnetic element) content in these tissues and fungi. ${ }^{17}$ Other renal fungal infections like candidiasis present as multiple microabscesses, papillary necrosis, and fungal balls within the collecting system whereas renal aspergilloma present as focal mass lesions. ${ }^{18}$

Though the suspicion of mucormycosis can be made with supportive clinical and imaging findings, a definitive diagnosis can be made only with histopathological findings. The treatment of renal mucormycosis is intravenous liposomal amphotericin B and emergency nephrectomy with debridement of nearby involved tissues. Despite treatment, the mortality rate associated with mucormycosis in general are very high averaging around $54 \%$ with the highest mortality rate of $96 \%$ for disseminated mucormycosis. ${ }^{7}$

Conflict of Interest

None declared.

\section{Acknowledgments}

The authors acknowledge all the departments involved in the management of the patient. The authors sincerely thank their mentors, colleagues, and staff personnel for helping with this case report.

\section{References}

1 WHO Coronavirus disease (COVID-19) Dashboard with vaccination data. Accessed July 30, 2021 at: https://www.covid19.who. int/

2 Salehi M, Ahmadikia K, Badali H, Khodavaisy S, Badali H, Khodavaisy S. Opportunistic fungal infections in the epidemic area of COVID-19; a clinical and diagnostic perspective from Iran. Mycopathologia 2020;185(04):607-611

3 Singh AK, Singh R, Joshi SR, Misra A. Mucormycosis in COVID-19: a systematic review of cases reported worldwide and in India. Diabetes Metab Syndr 2021;15(04):102146 
4 Spellberg B, Edwards J Jr, Ibrahim A. Novel perspectives on mucormycosis: pathophysiology, presentation, and management. Clin Microbiol Rev 2005;18(03):556-569

5 Choudhary GR, Aggarwal A, Jain V, Jena R. COVID-19 and fatal renal mucormycosis: contributory or coincidental? Indian J Urol 2021;37(03):270-273

6 Singh T, Chaudhari R, Gupta A. Renal artery thrombosis and mucormycosis in a COVID-19 patient. Indian J Urol 2021;37 (03):267-269

7 Roden MM, Zaoutis TE, Buchanan WL, et al. Epidemiology and outcome of zygomycosis: a review of 929 reported cases. Clin Infect Dis 2005;41(05):634-653

8 Agrawal R, Yeldandi A, Savas H, Parekh ND, Lombardi PJ, Hart EM. Pulmonary mucormycosis: risk factors, radiologic findings, and pathologic correlation. Radiographics 2020;40(03): 656-666

9 Mishra Y, Prashar M, Sharma D, Akash,Kumar VP, Tilak TVSVGK. Diabetes, COVID 19 and mucormycosis: clinical spectrum and outcome in a tertiary care medical center in Western India. Diabetes Metab Syndr 2021;15(04):102196

10 Garg D, Muthu V, Sehgal IS, et al. Coronavirus disease (Covid-19) associated mucormycosis (CAM): case report and systematic review of literature. Mycopathologia 2021;186(02):289-298
11 Ibrahim IM, Abdelmalek DH, Elshahat ME, Elfiky AA. COVID-19 spike-host cell receptor GRP78 binding site prediction. J Infect 2020;80(05):554-562

12 Baldin C, Ibrahim AS. Molecular mechanisms of mucormycosisthe bitter and the sweet. PLoS Pathog 2017;13(08):e1006408

13 Seoane PI, Taylor-Smith LM, Stirling D, et al. Viral infection triggers interferon-induced expulsion of live Cryptococcus neoformans by macrophages. PLoS Pathog 2020;16(02):e1008240

14 Gupta KL, Joshi K, Sud K, et al. Renal zygomycosis: an underdiagnosed cause of acute renal failure. Nephrol Dial Transplant 1999;14(11):2720-2725

15 Levy E, Bia MJ. Isolated renal mucormycosis: case report and review. J Am Soc Nephrol 1995;5(12):2014-2019

16 Keogh CF, Brown JA, Phillips P, Cooperberg PL. Renal mucormycosis in an AIDS patient: imaging features and pathologic correlation. AJR Am J Roentgenol 2003;180(05):1278-1280

17 Ranjan P, Naval R, Singh R, Gupta RK, Kapoor R, Sharma RK. Emerging role of radiological criteria for antemortem diagnosis of renal zygomycosis: an uncommon cause of acute renal failure. NDT Plus 2011;4(05):335-338

18 Orlowski HLP, McWilliams S, Mellnick VM, et al. Imaging spectrum of invasive fungal and fungal-like infections. Radiographics 2017;37(04):1119-1134 